\title{
On the Flexibility of a Transmuted Type I Generalized Half Logistic Distribution with Application
}

\author{
Adeyinka Femi Samuel*, Olapade Akintayo Kehinde \\ Department of Mathematsics, Obafemi Awolowo University, Ile-Ife, Nigeria \\ Email address: \\ fs.adeyinka247@gmail.com (A. F. Samuel), akolapad@oauife.edu.ng (O. A. Kehinde) \\ ${ }^{*}$ Corresponding author
}

\section{To cite this article:}

Adeyinka Femi Samuel, Olapade Akintayo Kehinde. On the Flexibility of a Transmuted Type I Generalized Half Logistic Distribution with Application. Engineering Mathematics. Vol. 3, No. 1, 2019, pp. 13-18. doi: 10.11648/j.engmath.20190301.14

Received: June 5, 2019; Accepted: July 5, 2019; Published: July 16, 2019

\begin{abstract}
In this article we transmute the type I half logistic distribution using quadratic rank transmutation map to develop a transmuted type I half logistic distribution. The quadratic rank transmutation map enables the introduction of extra parameter into its baseline distribution to enhance more flexibility in the analysis of data in various disciplines such as reliability analysis in engineering, survival analysis, medicine, biological sciences, actuarial science, finance and insurance. The mathematical properties such as moments, quantile, mean, median, variance, skewness and kurtosis of this distribution are discussed. The reliability and hazard functions of the transmuted type I half logistic distribution are obtained. The probability density functions of the minimum and maximum order statistics of the transmuted type I half logistic distribution are established and the relationships between the probability density functions of the minimum and maximum order statistics of the parent model and the probability density function of the transmuted type I half logistic distribution are considered. The parameter estimation is done by the method of maximum likelihood estimation. The flexibility of the model in statistical data analysis and its applicability is demonstrated by using it to fit relevant data. The study is concluded by demonstrating that the transmuted type I half logistic distribution has a better goodness of fit than its parent model. We hope this model will serve as an alternative to the existing ones in the literature in fitting positive real data.
\end{abstract}

Keywords: Half logistic Distribution, Reliability Function, Hazard Rate Function, Parameter Estimation, Order Statistics, Transmutation

\section{Introduction}

Half logistic distribution is a useful probability model in areas such as biostatistics, reliability analysis, survival analysis and other disciplines. This distribution has gained the attention of many researchers over the years.

The half logistic distribution which was studied by Balakrishnan [1] and Balakrishnan and Puthenpura [2] has the probability density function given by

$$
f(x)=\frac{2 e^{x}}{\left(1+e^{x}\right)^{2}}, x>0
$$

Olapade [3] generalized (1) above which he called the type I generalized half logistic distribution with probability density function given by

$$
g(x)=\frac{{ }_{2}^{\beta} \beta e^{x}}{\left(1+e^{x}\right)^{\beta+1}}, x>0, \beta>0 .
$$

Some mathematical properties and application of the model were presented in the paper.

This work intends to study the transmuted type I generalized half logistic distribution.

\section{Transmuted Type I Generalized Half Logistic Distribution}

If a random variable $X$ has the type I generalized half logistic distribution with probability density function (pdf) given in (2) and the cumulative distribution function (cdf) given by 


$$
G(x)=1-\frac{2^{\beta}}{\left(1+e^{x}\right)^{\beta}}, x>0, \beta>0 .
$$

The corresponding cdf of transmuted type I generalized half logistic distribution, using the quadratic rank transmutation map,

$$
F(x)=(1+\lambda) G(x)-\lambda G^{2}(x),|\lambda| \leq 1
$$

is given by

$$
F(x)=\frac{\left(1+e^{x}\right)^{2 \beta}-2^{\beta}\left\{(1-\lambda)\left(1+e^{x}\right)^{\beta}+2^{\beta} \lambda\right\}}{\left(1+e^{x}\right)^{2 \beta}}, x>0, \beta>0,|\lambda| \leq 1
$$

$ß=2.0$

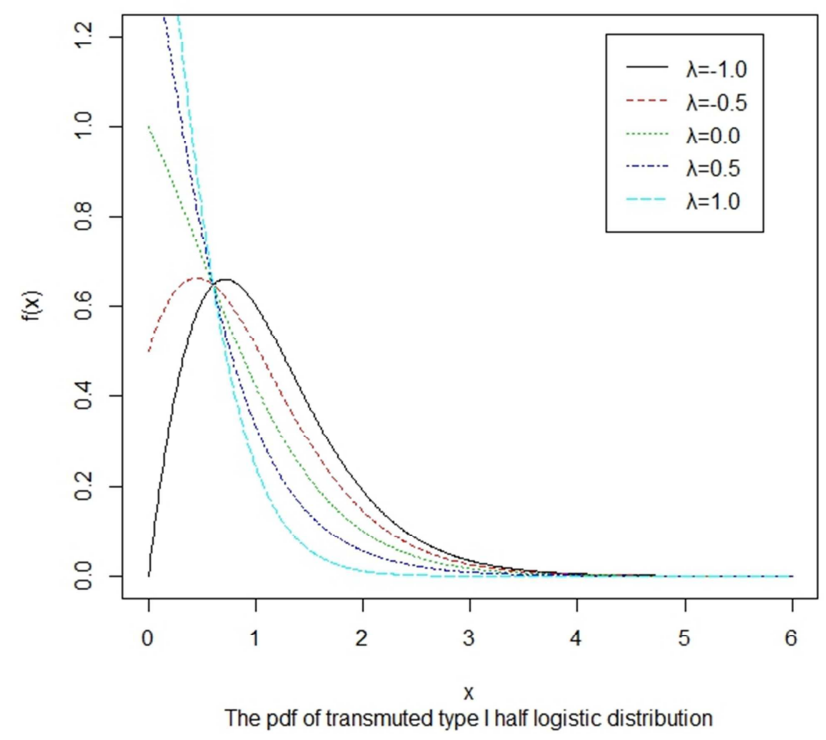

and the corresponding pdf is obtained by differentiating (5) with respect to $\mathrm{x}$ and it is given by

$$
f(x)=\frac{2^{\beta} \beta e^{x}\left\{(1-\lambda)\left(1+e^{x}\right)^{\beta}+2^{\beta+1} \lambda\right\}}{\left(1+e^{x}\right)^{2 \beta+1}}, x>0 .
$$

The probability density function in (6) is called transmuted type I generalized half logistic distribution. When $\lambda=0$, we have the pdf of the type I generalized half logistic distribution in (2).

Figure 1 below shows the graphs of pdf of the transmuted type I generalized half logistic distribution for different values of parameters $\beta$ and $\lambda$.

$ß=2.5$

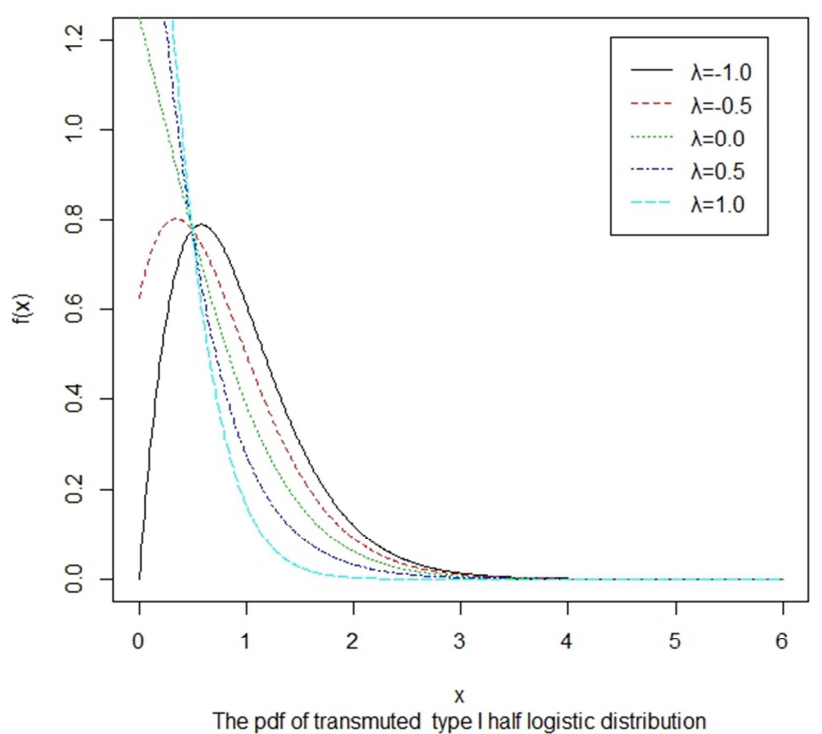

Figure 1. The graphs of pdf of transmuted type I generalized half logistic distribution.

\section{Moments and Quantiles}

The $k^{\text {th }}$ order moment of the transmuted type I generalized half logistic random variable $\mathrm{X}$, is given by

$$
E\left[X^{k}\right]=2^{\beta} \beta \int_{0}^{\infty} x^{k} e^{x} \frac{\left\{(1-\lambda)\left(1+e^{x}\right)^{\beta}+2^{\beta+1} \lambda\right\}}{\left(1+e^{x}\right)^{2 \beta+1}} d x .
$$

The result cannot be obtained explicitly hence it is obtained numerically using Maple software. Table 1 below shows the moments for various values of parameters $\beta$ and $\lambda$.

The $q^{\text {th }}$ quantile $x_{q}$ of the transmuted type I generalized half logistic distribution can be obtained from (5) as

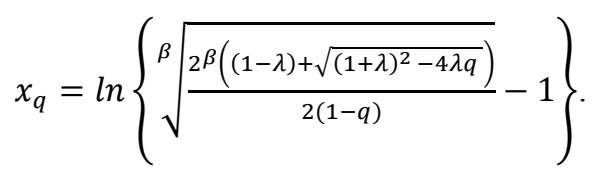

The median of the transmuted type I generalized half logistic distribution is obtained when $q=0.5$ in (8) to have

$$
x_{0.5}=\ln \left\{\sqrt[\beta]{2^{\beta}\left((1-\lambda)+\sqrt{1+\lambda^{2}}\right)}-1\right\} .
$$

Table 1. The Moments of the Transmuted Type I Generalized half logistic Distribution for selected values of parameter $\lambda$ when parameter $\beta=5.0$.

\begin{tabular}{llllll}
\hline $\boldsymbol{\lambda}$ & $\boldsymbol{E}[\boldsymbol{X}]$ & $\boldsymbol{E}\left[\boldsymbol{X}^{\mathbf{2}}\right]$ & $\boldsymbol{E}\left[\boldsymbol{X}^{\mathbf{3}}\right]$ & $\boldsymbol{E}\left[\boldsymbol{X}^{\mathbf{4}}\right]$ & $\operatorname{Var}[\boldsymbol{X}]$ \\
\hline-1.0 & 0.5105 & 0.3730 & 0.3501 & 0.3984 & 0.1124 \\
-0.75 & 0.4697 & 0.3343 & 0.3102 & 0.3511 & 0.1137 \\
-0.5 & 0.4289 & 0.2956 & 0.2703 & 0.3037 & 0.1116 \\
-0.25 & 0.3881 & 0.2570 & 0.2305 & 0.2563 & 0.1064 \\
0.0 & 0.3474 & 0.2183 & 0.1906 & 0.2090 & 0.0976 \\
0.25 & 0.3066 & 0.1796 & 0.1507 & 0.1615 & 0.0856 \\
0.5 & 0.2658 & 0.1409 & 0.1109 & 0.1141 & 0.0703 \\
0.75 & 0.2251 & 0.1022 & 0.0710 & 0.0668 & 0.0515 \\
1.0 & 0.1843 & 0.0636 & 0.0311 & 0.0194 & 0.0296 \\
\hline
\end{tabular}

\section{The Skewness and Kurtosis of the Transmuted Type I Generalized half logistic Distribution}

The coefficient of skewness of the transmuted type I generalized half logistic distribution is denoted by $\beta_{1}$ such that

$$
\beta_{1}=\frac{\mu_{3}^{2}}{\mu_{2}^{3}}
$$


Where $\mu_{2}=\operatorname{Var}[X]=E\left[X^{2}\right]-(E[X])^{2}$ and $\mu_{3}=$ $E\left[X^{3}\right]-3 E\left[X^{2}\right] E[X]+2(E[X])^{3}$.

The coefficient of kurtosis of the transmuted type I generalized half logistic distribution is denoted by $\beta_{2}$ such that

$$
\beta_{2}=\frac{\mu_{4}}{\mu_{2}^{2}}
$$

where $\quad \mu_{4}=E\left[X^{4}\right]-4 E\left[X^{3}\right] E[X]+6 E\left[X^{2}\right](E[X])^{2}-$ $3(E[X])^{4}$

The skewness and kurtosis of the transmuted type I generalized half logistic distribution for selected values of parameter $\lambda$ when parameter $\beta=5.0$ is shown in Table 2 below.

Table 2. The skewness and kurtosis of the Transmuted Type I Generalized half logistic Distribution for selected values of parameter $\lambda$ when parameter $\beta=5.0$.

\begin{tabular}{lllll}
\hline $\boldsymbol{\lambda}$ & $\boldsymbol{\mu}_{\mathbf{3}}$ & $\boldsymbol{\mu}_{\mathbf{4}}$ & $\boldsymbol{S k w e n e s s}\left(\boldsymbol{\beta}_{\mathbf{1}}\right)$ & $\operatorname{Kurtosis}\left(\boldsymbol{\beta}_{2}\right)$ \\
\hline-1.0 & 0.3994 & 1.2197 & 1.35 & 5.07 \\
-0.75 & 0.4115 & 1.2562 & 1.36 & 5.03 \\
-0.5 & 0.4246 & 1.2570 & 1.50 & 5.14 \\
-0.25 & 0.4277 & 1.2300 & 1.72 & 5.48 \\
0 & 0.4222 & 1.1386 & 2.13 & 5.94 \\
0.25 & 0.3911 & 1.0031 & 2.65 & 6.72 \\
0.5 & 0.3286 & 0.8026 & 3.30 & 7.83 \\
0.75 & 0.2278 & 0.5151 & 3.83 & 9.06 \\
1.0 & 0.0801 & 0.1201 & 2.26 & 6.00 \\
\hline
\end{tabular}

\section{Random Number Generation and Parameter Estimation}

Using the method of inversion we can generate random numbers from the transmuted type I generalized half logistic distribution as

$$
\frac{\left(1+e^{x}\right)^{2 \beta}-2^{\beta}\left\{(1-\lambda)\left(1+e^{x}\right)^{\beta}+2^{\beta} \lambda\right\}}{\left(1+e^{x}\right)^{2 \beta}}=u
$$

where $u \sim U(0,1)$. After simplifying (10) we have

$$
x=\ln \left\{\sqrt[\beta]{\frac{2^{\beta\left((1-\lambda)+\sqrt{(1+\lambda)^{2}-4 \lambda u}\right)}}{2(1-u)}}-1\right\} .
$$

Equation (11) can be used to generate random numbers when the parameters $\beta$ and $\lambda$ are known.

\section{Parameter Estimation}

The maximum likelihood estimates of the parameters $\beta$ and $\lambda$ that are inherent within the transmuted type I generalized half logistic distribution function are given by the following:

Let $X_{1}, X_{2}, \ldots X_{n}$ be a sample of size $\mathrm{n}$ from a generalized transmuted half logistic distribution. The likelihood function is given by

$$
L=2^{\beta n} \beta^{n} e^{\sum_{i=1}^{n} x_{i}} \prod_{i=1}^{n}\left\{(1-\lambda)\left(1+e^{x_{i}}\right)^{\beta}+2^{\beta+1} \lambda\right\} / \prod_{i=1}^{n}\left(1+e^{x_{i}}\right)^{2 \beta+1}
$$

Hence the log-likelihood function becomes

$$
\ln L=\beta n \ln 2+n \ln \beta+\sum_{i=1}^{n} x_{i}+\sum_{i=1}^{n} \ln \left\{(1-\lambda)\left(1+e^{x_{i}}\right)^{\beta}+2^{\beta+1} \lambda\right\}-(2 \beta+1) \sum_{i=1}^{n} \ln \left(1+e^{x_{i}}\right) .
$$

By differentiating (13) with respect to parameters $\beta$ and $\lambda$ and equating the result to zero we have

$$
\frac{\partial \ln L}{\partial \lambda}=\sum_{i=1}^{n} \frac{2^{\beta+1}-\left(1+e^{x_{i}}\right)^{\beta}}{\left[(1-\lambda)\left(1+e^{x_{i}}\right)^{\beta}+2^{\beta+1} \lambda\right]}=0 \frac{\partial \ln L}{\partial \beta}=n \ln 2+\frac{n}{\beta}-2 \sum_{i=1}^{n} \ln \left(1+e^{x_{i}}\right)+\sum_{i=1}^{n} \frac{\beta(1-\lambda)\left(1+e^{x_{i}}\right)^{\beta-1}+2^{\beta+1} \lambda \ln 2}{\left[(1-\lambda)\left(1+e^{x_{i}}\right)^{\beta}+2^{\beta+1} \lambda\right]}=0
$$

The maximum likelihood estimator $\hat{\theta}=(\hat{\beta}, \hat{\lambda})^{\prime}$ of parameters $\theta=(\beta, \lambda)^{\prime}$ can be obtained by solving this nonlinear system of equations. It is usually more convenient to use non-linear optimization algorithms such as quasi-Newton algorithm to numerically maximize the log-likelihood function in (13).

\section{Reliability Analysis}

The survival function, also known as the reliability function in engineering, is the characteristic of the explanatory variable that maps a set of events, usually associated with mortality or failure of some system unto time. It is the probability that a system will survive beyond a specified time.

The transmuted type I generalized half logistic distribution can be a useful model to characterize failure time of a given system because of the analytical structure. The reliability function $R(t)$, which is the probability of an item not failing prior to some time $t$, is defined by $R(t)=1-F(t)$. The reliability function of the transmuted type I generalized half logistic distribution is given by

$$
R(t)=\frac{2^{\beta}\left\{(1-\lambda)\left(1+e^{t}\right)^{\beta}+2^{\beta} \lambda\right\}}{\left(1+e^{t}\right)^{2 \beta}} .
$$

Figure 2(ii) below illustrates the reliability behaviour of transmuted type I generalized half logistic distribution as the value of parameter $\lambda$ varies from -1.0 to 1.0

The other characteristics of interest of a random variable is the hazard rate function also known as instantaneous failure rate defined by

$$
h(t)=\frac{f(t)}{1-F(t)}
$$

this is an important quantity characterizing life phenomenon. It can be loosely interpreted as the conditional probability of failure, given it has survived to the time $t$. The hazard rate 
function of transmuted type I generalized half logistic distribution is given by

$$
h(t)=\frac{\beta e^{t}\left\{(1-\lambda)\left(1+e^{t}\right)^{\beta}+2^{\beta+1} \lambda\right\}}{\left(1+e^{t}\right)\left[(1-\lambda)\left(1+e^{t}\right)^{\beta}+2^{\beta} \lambda\right]} .
$$

Figure 2(ii) below illustrates the behavior of hazard rate function of transmuted type I generalized half logistic distribution for the selected values of parameter $\lambda$.

The fundamental relationship between reliability function $R(t)$ and cumulative hazard function $H(t)$ is given by

$B=2.0$

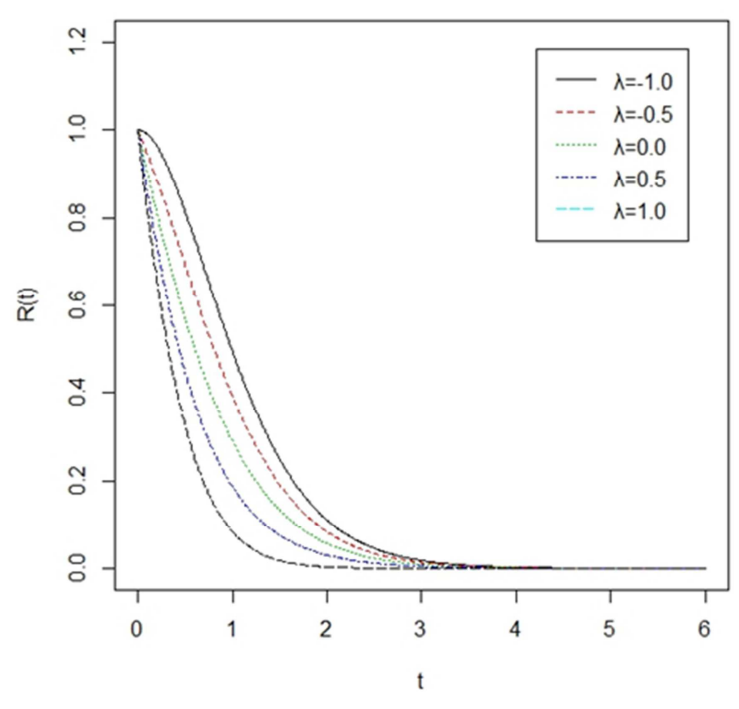

(i)
$H(t)=-\ln R(t)$. The cumulative hazard function of the type I generalized half logistic distribution is given by

$$
H(t)=\int_{0}^{t} h(x) d x=\ln \left\{\frac{\left(1+e^{t}\right)^{2 \beta}}{2^{\beta}\left\{(1-\lambda)\left(1+e^{t}\right)^{\beta}+2^{\beta} \lambda\right\}}\right\} .
$$

It is observed that

i) $H(t)$ is a non-decreasing for all $t \geq 0$

ii) $H(0)=0$

iii) $\lim _{t \rightarrow \infty} H(t)=\infty$.

\section{$ß=2.0$}

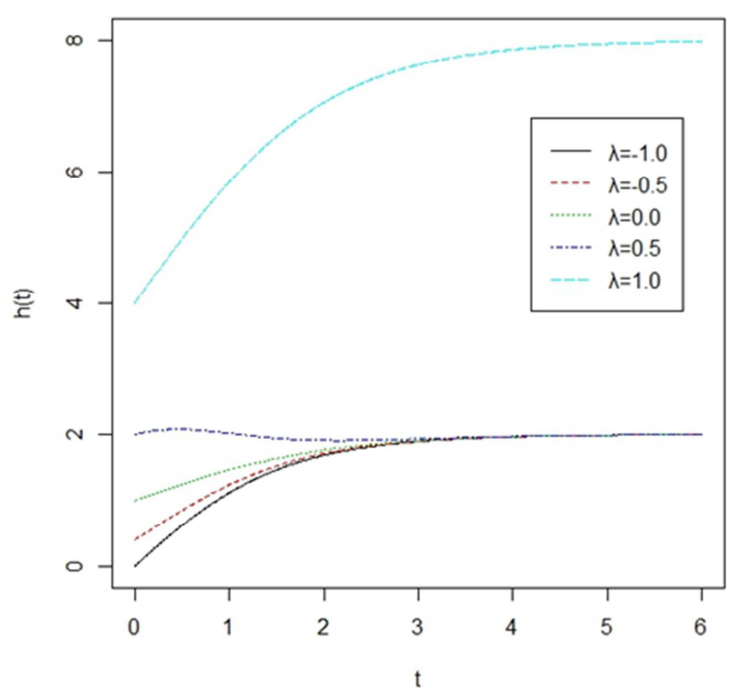

(ii)

Figure 2. The graphs of reliability function and hazard function of transmuted type I generalized half logistic distribution.

Order Statistics

We know that if $X_{(1)} \leq X_{(2)} \leq \cdots \leq X_{(n)}$ denotes the order statistics of a random sample $X_{1}, X_{2} \ldots X_{n}$ from a continuous population with cdf $F_{X}(x)$ and pdf $f_{X}(x)$, David [4] gave the probability density function of $X_{(r)}$ as

$$
f_{X_{(r)}}(x)=\frac{1}{B(r, n-r+1)}[F(x)]^{r-1}[1-F(x)]^{n-r} f(x)
$$

where $r=1,2, \ldots n$.

We have from (2) and (3) the pdf of the $r^{\text {th }}$ order type I generalized half logistic random variable $X_{(r)}$ given by

$$
g_{X_{(r)}}(x)=\frac{2^{\beta(n-r+1)} \beta e^{x}\left\{\left(1+e^{x}\right)^{\beta}-2^{\beta}\right\}^{r-1}}{B(r, n-r+1)\left(1+e^{x}\right)^{\beta n+1}}
$$

Therefore the pdf of the $n t h$ order type I generalized half logistic statistic $X_{(n)}$ is given by

$$
g_{X_{(n)}}(x)=\frac{2^{\beta} \beta n e^{x}\left\{\left(1+e^{x}\right)^{\beta}-2^{\beta}\right\}^{n-1}}{\left(1+e^{x}\right)^{\beta n+1}}
$$

and the pdf of the $1^{\text {st }}$ order type I generalized half logistic statistic $X_{(1)}$ is given by

$$
g_{X_{(1)}}(x)=\frac{2^{\beta n} \beta n e^{x}}{\left(1+e^{x}\right)^{\beta n+1}}
$$

Note that in a particular case of $n=2,(15)$ yields

$$
g_{X_{(1)}}(x)=\frac{2^{2 \beta+1} \beta e^{x}\left\{\left(1+e^{x}\right)^{\beta}-2^{\beta}\right\}}{\left(1+e^{x}\right)^{2 \beta+1}}
$$

and (16) yields

$$
g_{X_{(2)}}(x)=\frac{2^{2 \beta+1} \beta e^{x}}{\left(1+e^{x}\right)^{2 \beta+1}}
$$

It can be observed that $\min \left(X_{1}, X_{2}\right)$ and $\max \left(X_{1}, X_{2}\right)$ are special cases of (5) for $\lambda=1$ and $\lambda=-1$ respectively.

Now we provide the distribution of the order statistics for the transmuted type I generalized half logistic random variable. The pdf of the $r^{t h}$ order statistics for the transmuted type I generalized half logistic distribution is given in (13).

We have from (4) and (5) the pdf of the $r^{\text {th }}$ order statistics for the generalized transmuted half logistic distribution given by

$$
f_{X_{(r)}}(x)=\frac{2^{\beta} \beta e^{x}\left\{(1-\lambda)\left(1+e^{x}\right)^{\beta}+2^{\beta+1} \lambda\right\}\left\{\left(1+e^{x}\right)^{2 \beta}-2^{\beta}(1-\lambda)\left(1+e^{x}\right)^{\beta}-2^{2 \beta} \lambda\right\}^{r-1}\left\{2^{\beta}(1-\lambda)\left(1+e^{x}\right)^{\beta}+2^{2 \beta} \lambda\right\}^{n-r}}{B(r, n-r+1)\left(1+e^{x}\right)^{2 \beta n+1}}
$$


Therefore the pdf of the largest order statistic $X_{(n)}$ is given by

$$
f_{X_{(n)}}(x)=\frac{2^{\beta} \beta n e^{x}\left\{(1-\lambda)\left(1+e^{x}\right)^{\beta}+2^{\beta+1} \lambda\right\}\left\{\left(1+e^{x}\right)^{2 \beta}-2^{\beta}(1-\lambda)\left(1+e^{x}\right)^{\beta}-2^{2 \beta} \lambda\right\}^{n-1}}{\left(1+e^{x}\right)^{2 \beta n+1}}
$$

and the pdf of the smallest order statistic $X_{(1)}$ is given by

$$
f_{X_{(1)}}(x)=\frac{2^{\beta} \beta n e^{x}\left\{(1-\lambda)\left(1+e^{x}\right)^{\beta}+2^{\beta+1} \lambda\right\}\left\{2^{\beta}(1-\lambda)\left(1+e^{x}\right)^{\beta}+2^{2 \beta} \lambda\right\}^{n-1}}{\left(1+e^{x}\right)^{2 \beta n+1}}
$$

\section{Application}

The data represents the survival time of 72 Guinea Pigs infected with virulent tubercle bacilli. The data set is obtained from the work of Usman, Haq and Talib [5].

The data are as follows:

$0.1,0.33,0.44,0.56,0.59,0.72,0.74,0.77,0.92,0.93$, $0.96,1.00,1.00,1.02,1.05,1.07,0.7,0.08,1.08,1.08,1.09$, $1.12,1.13,1.15,1.16,1.20,1.21,1.22,1.22,1.24,1.3,1.34$, $1.36,1.39,1.44,1.46,1.53,1.59,1.60,1.63,1.63,1.68,1.71$, $1.72,1.76,1.83,1.95,1.96,1.97,2.02,2.13,2.15,2.16,2.22$, $2.30,2.31,2.40,2.45,2.51,2.53,2.54,2.54,2.78,2.93,3.27$, $3.42,3.47,3.61,4.02,4.32,4.58,5.55$.

A quasi Newton algorithm was implemented in R package and the performances of the models are shown in Table 3 . Akaike Information criterion (AIC), Corrected Akaike Information criterion (AICC) and Bayesian Information criterion (BIC) were respectively used to compare the performance of type I generalized half logistic distribution (THL) to its parent model (HL) in (2).

$$
\begin{gathered}
A I C=2 k-2 L L \\
A I C C=A I C+\frac{2 k(k+1)}{n-k-1}
\end{gathered}
$$

And

$$
B I C=2 \log (n)-2 L L
$$

Where $\mathrm{k}$ is the number of parameters in the model, $\mathrm{n}$ is the sample size and LL is the maximized value of log likelihood function.

Table 3. Performance of the models.

\begin{tabular}{llllll}
\hline Model & Estimates & -LL & AIC & AICC & BIC \\
\hline THL & $\beta=3.6017$ & 142.672 & 289.344 & 289.518 & 289.059 \\
HL & $\lambda=0.8989$ & & & & \\
& $\beta=3.2751$ & 147.135 & 296.270 & 296.326 & 296.127 \\
\hline
\end{tabular}

It is observed that the transmuted type I generalized half logistic distribution (THL) performs better than its parent model (HL) in (2).

\section{Conclusion}

In this article, we have introduced a new generalization of the type I generalized half logistic distribution called transmuted type I generalized half logistic distribution. The distribution which is generalized by using the quadratic rank transmutation map. Some mathematical properties along with estimation issues are addressed. The hazard rate function and reliability behaviour of the transmuted type I generalized half logistic distribution shows that the subject distribution can be used to model positive data that follow the type I generalized half logistic model. Further studies can be done in the area of applicability of the model in various disciplines. We expect that this study will serve as a reference and help to advance future research in this area and other related fields.

\section{References}

[1] Balakrishnan, N. (1985). Order statistics from half logistic distribution. Journal of Statistical Computation and Simulation. 20, 287-309.

[2] Balakrishnan, N., and Puthenpura, S. (1986). Best linear unbiased estimators of location and scale parameters of the half logistic distribution. Journal of Statistical Computation and Simulation. Vol 4. Pp 193-204.

[3] Olapade, A. K. (2014), The Type I Generalized Half Logistic Distribution. JIRSS. Vol. 13, No. 1, pp 69-82.

[4] David, H. A. (1970) Order Statistics. New York: Wiley Interscience series.

[5] Usman, R. M, Haq, M. A and Talib, J (2017). Kumaraswamy Half-Logistic Distribution: Properties and Applications. Journal of Statistics Applications and Probability. No 3, 597609 .

[6] Aryal, G. R, and Tsokos, C. P. (2009). On the transmuted extreme value distribution with application. Nonlinear Analysis: Theory, Methods and Application. 71 (12), el401el407.

[7] Aryal, G. R, and Tsokos, C. P. (2011). Transmuted Weilbull distribution: A generalization of Weilbull probability distribution. European Journal of Pure and Applied Mathematics. 4 (2), 89-102.

[8] Bjerkedal, T (1960). Acquisition of Resistance in Guinea Pigs infected with Different Doses of Virulent Tubercle Bacilli, American Journal of Hygiene, 72, 130-148.

[9] Haq, M. A, (2016). Kumaraswamy Exponentiated Inverse Rayleigh Distribution, Mathematical Theory and Modeling, 6, 93-104.

[10] Merovci, F., Alizadeh, M., and Hamedani, G. (2016). Another Generalized Transmuted Family of Distributions: Properties and Applications. Austrian Journal of Statistics. 45, 71-93.

[11] Merovci, F. (2014). Transmuted Generalized Rayleigh Distribution. Journal of Statistics Applications and Probability. $3(1), 9-20$. 
[12] Merovci, F., Elbatal, I. (2014). Transmuted Lindley-geometric Distribution and its Applications. Journal of Statistics Applications and Probability. 3 (1), 77-91.

[13] Merovci, F., Puka, L. (2014). Transmuted Pareto Distribution. Probstat. 7, 1-11.

[14] Olapade, A. K. (2003), On Characterizations of the Half Logistic Distri-bution. InterStat, February Issue, 2, http://interstat.stat.vt.edu/InterStat/ARTICLES/2003articles/F 06002.pdf.
[15] Rahman M. M, Al-Zahrani B, Shahbaz M. Q (2018). A general transmuted family of distributions. Pak J Stat Oper Res 14: 451-469.

[16] Shaw, W. T, and Buckley, I. R. (2009). Alchemy of Probability Distributions: Beyond Gram-Charlier and Cornish -Fisher Expansions, and Skewed- kurtotic Normal Distribution from a Rank Transmutation Map. arxivpreprint arxiv: 0901.0434. 